\title{
Editorial: Ethnopharmacological Responses to the Coronavirus Disease 2019 Pandemic
}

\author{
Jia-bo Wang ${ }^{1}$, Adolfo Andrade-Cetto ${ }^{2}$, Javier Echeverria ${ }^{3}$, Jon Wardle ${ }^{4}$, Hung-Rong Yen ${ }^{5}$ \\ and Michael Heinrich ${ }^{6 *}$ \\ ${ }^{1}$ School of Traditional Chinese Medicine, Capital Medical University, Beijing, China, ${ }^{2}$ Laboratorio de Etnofarmacología, Facultad \\ de Ciencias, Universidad Nacional Autónoma de México, Mexico City, Mexico, ${ }^{3}$ Departamento de Ciencias del Ambiente, \\ Facultad de Química y Biología, Universidad de Santiago de Chile, Santiago, Chile, ${ }^{4}$ National Centre for Naturopathic Medicine, \\ Southern Cross University, Lismore, NSW, Australia, ${ }^{5}$ Chinese Medicine Research Center and College of Chinese Medicine, \\ China Medical University, Taichung, Taiwan, ${ }^{6}$ Research Group "Pharmacognosy and Phytotherapy", UCL School of Pharmacy, \\ University of London, London, United Kingdom
}

Keywords: COVID-19, ethnopharmacology, traditional medicine, adjuvant therapies, clinical study, systematic review, mechanism study

Editorial on the Research Topic

Ethnopharmacological Responses to the Coronavirus Disease 2019 Pandemic

\section{OPEN ACCESS}

Edited by:

Thomas Brendler,

PlantaPhile, Collingswood,

United States

Reviewed by:

Elizabeth Mary Williamson,

University of Reading, United Kingdom

${ }^{*}$ Correspondence:

Michael Heinrich

m.heinrich@ucl.ac.uk

Specialty section: This article was submitted to

Ethnopharmacology,

a section of the journal

Frontiers in Pharmacology

Received: 20 October 2021 Accepted: 10 November 2021 Published: 03 December 2021

Citation:

Wang J-b Andrade-Cetto A, Echeverria J, Wardle J, Yen H-R and Heinrich M (2021) Editorial: Ethnopharmacological Responses to the Coronavirus Disease 2019 Pandemic

Front. Pharmacol. 12:798674. doi: 10.3389/fphar.2021.798674

\section{INTRODUCTION}

The pandemic of coronavirus disease 2019 (COVID-19), caused by severe acute respiratory syndrome coronavirus 2 (SARS-CoV-2) infection, has led to severe impact on health globally. There is no effective therapy for SARS-CoV-2 infection except the preventive effects of a vaccine. Several medicines (PF07321332, EXO-CD24, BRII-196, and BRII-198 etc.) against SARS-CoV-2 infection are under development; but the clinical efficacy of these medicines has not been evaluated rigorously. The outputs and prices make them less accessible, especially in low-and middle-income countries (LMIC). The lack of effective medicines has led to the very high global demand for various forms of traditional medicines (TM) as a potential treatment option for COVID-19. Simultaneously, the use of herbal medicines and supplements (especially those with anti-infective and immunomodulatory effects as well as those used as a supportive therapy) has increased dramatically as a part of adjuvant therapy in economically developed countries, which is often not reported to health care professionals (Bhamra et al. 2021; Smith et al.). In many LMIC regions including in China, India, South Korea, Thailand, the Americas and Africa, TMs are accessible and widely used and numerous TM therapies have started to be investigated as potential treatments. Some of the claims made, however, are very high and seem implausible. We reiterate that these TM treatments should be seen as a part of a broader package of adjuvant and symptomatic treatments and that scientists have a specific responsibility to ascertain a balanced and critical assessment of the evidence as well as the gaps (Heinrich 2010).

For example, traditional African medicine (TAM), traditional Chinese medicine (TCM), traditional Indian medicine (TIM), and traditional Persian medicine (TPM) have shown unique potentials to be used generally as a therapy against COVID-19. This is linked to reported immunomodulatory, anti-inflammatory, antiviral, antioxidant, antihistamine, and bronchodilator effects (Bahramsoltani and Rahimi; Ahmad et al.; Attah et al.; Mosleh et al.; Saggam et al.). This widespread use and the existing preliminary evidence highlight the need of 

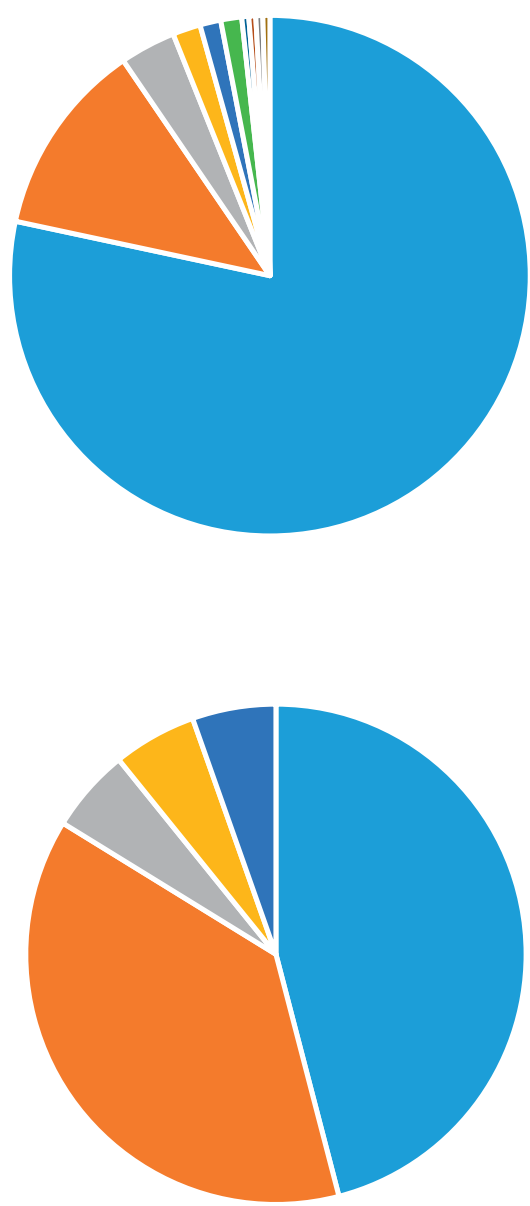

- Traditional Chinese Medicine

- Herbal medicine - unassigned

- Ayurveda

- Traditional African medicine

- Unani

- Traditional Persian medicine

- Kampo medicine

- Bangladeshi Medicine

- Indonesian traditions

- Turkish local medicine

- Traditional Chinese Medicine

- Herbal medicine - unassigned

- Ayurveda

- Traditional African medicine

- Traditional Persian medicine

FIGURE 1 | COVID-19 papers focusing on different local and traditional medical systems (TMs) (A) in PubMed. and (B) represented in this research topic.

clinical evidence from rigorous randomized controlled trials (RCTs) and meta-analysis, as well as in vitro and in vivo studies to assess the efficacy and the mechanisms of TMs against COVID-19.

This research topic is a collection of 37 articles focusing on the adjuvant effects, pharmacological characteristics, efficacy and safety of TMs, and the potential mechanisms against COVID19 of TM formulations, medicinal plants, and active components.

\section{USAGE OF TMS IN THE TREATMENT OF COVID-19 AND RELATED SYMPTOMS}

To get a view of the usage of TMs in the treatment of COVID-19, we searched PubMed to illustrate the proportion of different kinds of TMs with the time range from January 1, 2019 to August 12, 2021. In total, 231 studies of TMs on COVID-19 were retrieved. Among these studies, most abundant were studies on TCM (nearly $80 \% ; 181 / 231$ ); followed by studies which could not be classified into a specific geographical region or traditional medicine system (28 studies) (cf. also Brendler et al., 2021). The rest of the studies covered traditional medicine systems Ayurveda (Indian medicine), traditional, African, Unani, Kampo, and medicines from Bangladesh, Iran, Indonesia and Turkey (Figure 1A) (Ai et al., 2020; Ayatollahi et al., 2021; Benarba and Pandiella, 2020; Chen et al., 2021; Fakhri et al., 2021; Ge and He, 2020; Jha et al., 2021; Kim, 2021; Kalhori et al., 2021; Lem et al., 2021; Li et al., 2020; Lim et al., 2021; Liu et al., 2020; Luo et al., 2020; Majnooni et al., 2020; Ma et al., 2021; Mandal et al., 2021; Peter et al., 2021; Qiu et al., 2020; Remali and Aizat, 2021; Ren et al., 2021; Silveira et al., 2020; Spicer, 2021; Verma et al., 2020; Wu et al., 2020; Wu et al., 2021; Yang et al., 2020; Zhang et al., 2020; Zhou et al., 2020).

This research topic includes research on TCM, herbal medicine generally, Ayurvedic medicine, African, and Iranian medical traditions (Figure 1B), with TCM studies accounting for nearly 50\% (17/37).

\section{EVIDENCE-BASED MEDICINE OF TM STUDIES IN THE TREATMENT OF COVID-19}

Rigorous assessment on the level of evidence is of critical importance to guide future research and clinical management 


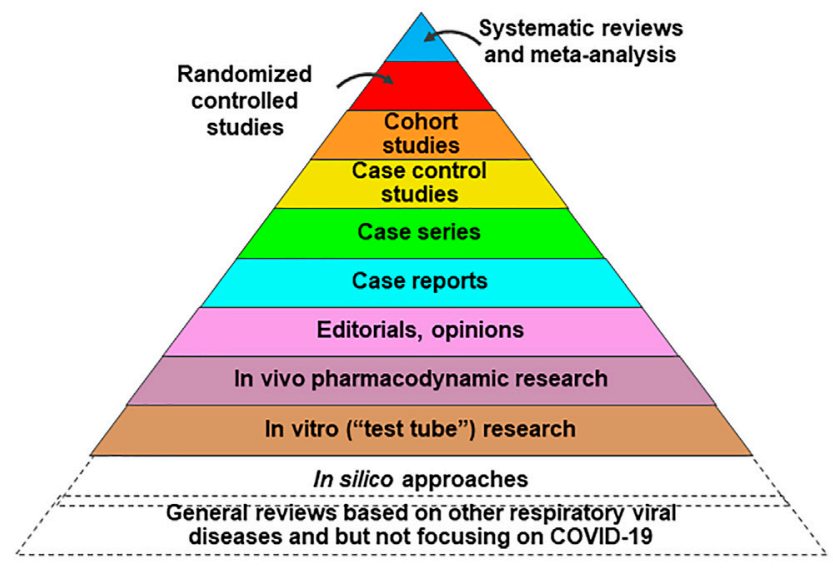

\begin{tabular}{|cc|} 
PubMed & $\begin{array}{c}\text { This Research } \\
\text { Topic }\end{array}$ \\
\hline 21 & 3 \\
\hline 12 & 0 \\
\hline 8 & 0 \\
\hline 14 & 1 \\
\hline 5 & 0 \\
\hline 4 & 0 \\
\hline 78 & 18 \\
\hline 5 & 3 \\
\hline 6 & 2 \\
\hline 58 & 4 \\
\hline 20 & 6 \\
\hline 231 & 37 \\
\hline
\end{tabular}

FIGURE 2 | Publication on TM associated with COVID-19.

on COVID-19 by TMs. Looking at the top level of evidence regarding the study of TMs on COVID-19 (Figure 2), in PubMed there are 21 systematic reviews (SRs) and meta-analyses and 3 SRs in this research topic. In PubMed 12 randomized controlled trials (RCTs) are recorded, and 11 of these RCTs evaluated the efficacy and safety of TCM in the treatment of COVID-19 and the other RCT (published on Frontiers in Pharmacology but not this research topic), tested oral use of a combined curcumin and piperine tablet, which are commonly used in India. We noted that we have no RCT papers but three highquality SRs in this research topic. Two of these three SRs performed meta-analysis and synthesized the current evidence to evaluate the clinical and safety of TCM intervention in the treatment of COVID-19. Beyond these RCT studies and meta-analysis, however, we can see that the majority of TMs-related COVID-19 studies indicate a relatively low level of evidence. There remains a dearth of original research on this topic, and those opinion papers or descriptive reviews have much overlap of ideas and cited references resulting in the risk of a limited scientific impact. With the limitation of good in vivo and in vitro SARS-Cov-2 models, we found a very limited body of scientific evidence. However, we found many in silico papers using molecular docking or network pharmacology that offer hypothetic leads based on TMs. Such non-validated in silico studies on TMs are, without associated in vivo, in vitro or clinical evidence, of limited relevance for informing clinical practice in COVID19 and can easily be misleading. Some review papers have been written based on experiences with other respiratory viral diseases but not based on COVID-19, specifically. These review papers were usually published in the early period of the pandemic, with a very limited knowledge regarding to COVID-19 at the time. These reviews may inspire new ideas for future studies on COVID-19, or of future management of a pandemic, considering the potential similarities of corona viruses and common courses of respiratory diseases and inflammatory responses. For instance, TCM treatments were broadly used and played an important role in other respiratory diseases, such as the severe acute respiratory syndrome (SARS) in 2003, the Influenza A (H1N1) in 2009, the Middle East Respiratory Syndrome (MERS) in 2012, and H7N9 avian influenza in 2013 (Xi et al.; Zhuang et al.), which may have some inspirations to understand the treatment of COVID-19. Collectively, although there is a growing body of publications on TMs against COVID-19, we need to be aware that we still lack high-quality clinical evidence, which highlights the importance of future clinical studies.

\section{EMPHASIS ON THE CLINICAL EVIDENCES OF TMS IN THE TREATMENT OF COVID-19}

Two SRs in this research topic evaluated the clinical efficacy of TCM in the treatment of COVID-19 and concluded that the interventions were safe in COVID-19 patients (Liang et al.; Wang et al.). One of these illustrated that TCM in combination with conventional therapy was better than conventional therapy alone. The purported beneficial effects included increasing the recovery rate of main symptoms of cough and fatigue, shortening the duration of main symptoms of fever, cough and fatigue, but were not suggestive of an increase the recovery rate of main symptoms of fever (Liang et al.). Another SR and meta-analysis focusing on low-risk-of-bias RCTs showed moderate confidence that compare to routine treatment alone, TCM plus routine treatment could reduce the incidence of unfavourable events of clinical deterioration, acute respiratory distress syndrome (ARDS), mechanical ventilation, and death). However, the treatment was not found to reduce the level of positive tests using the SARSCoV-2 nucleic acid test, and on chest X-ray images could 
shows improvements based (Wang et al.). Considering ARDS is the most common complications of COVID-19 (about 7. $4-41.8 \%$ of COVID-19 patients developed ARDS), as well as one of the most dangerous (the mortality rate of COVID-19 patients with ARDS was 30.4-52.4\%), the potentially favourable effect of specific TCM formula may indicate significant merit in further mechanistic studies (Wang et al.).

\section{PERSPECTIVES}

TMs have been used extensively in preventing and treating COVID-19 worldwide and in this short period a significant number papers have been published including a considerable share in this RT. These publications attracted considerable attention and have impacted on the fast-evolving discussion about the use of TMs. A wider debate is about the future role of TMs. Some countries including Thailand and the PR China have embraced TM as a potential strategy for ongoing treatment and/or prevention of COVID-19. In some cases, very strong claims about what can be achieved have been made, and these too may warrant further research attention. In many countries, however, the use of TMs as a strategy for COVID-19 is not accepted, resulting in such treatments becoming limited to overthe-counter self-treatment, often in unregulated or informal settings. An example is the dramatic rise of elderberry-based products (Sambucus nigra L.) sold in the United States in 2020 (Smith et al., 2021). Here the systematic assessment of opportunities to use such treatments as adjuvants and their appropriate role in the self-management of respiratory conditions more generally needs to be developed further. However, we should keep in mind to the lack of high-quality evidence-based TM-based treatments of COVID-19. More

\section{REFERENCES}

Adeloye, D., Elneima, O., Daines, L., Poinasamy, K., Quint, J. K., Walker, S., et al. (2021). The Long-Term Sequelae of COVID-19: an International Consensus on Research Priorities for Patients with Pre-existing and NewOnset Airways Disease. Lancet Respir. Med., 34416191. doi:10.1016/ S2213-2600(21)00286-1

Ai, Z., Zhou, S., Li, W., Wang, M., Wang, L., Hu, G., et al. (2020). "Fei Yan No. 1" as a Combined Treatment for COVID-19: An Efficacy and Potential Mechanistic Study. Front. Pharmacol. 11, 581277. doi:10.3389/fphar.2020.581277

Ayatollahi, S. A., Sharifi-Rad, J., Tsouh Fokou, P. V., Mahady, G. B., Ansar Rasul Suleria, H., Krishna Kapuganti, S., et al. (2021). Naturally Occurring Bioactives as Antivirals: Emphasis on Coronavirus Infection. Front. Pharmacol. 12, 575877. doi:10.3389/fphar.2021.575877

Bao, L., Deng, W., Huang, B., Gao, H., Liu, J., Ren, L., et al. (2020). The Pathogenicity of SARS-CoV-2 in hACE2 Transgenic Mice. Nature 583, 830-833. doi:10.1038/s41586-020-2312-y

Benarba, B., and Pandiella, A. (2020). Medicinal Plants as Sources of Active Molecules against COVID-19. Front. Pharmacol. 11, 1189. doi:10.3389/ fphar.2020.01189

Bhamra, S. K., Parmar, J., and Heinrich, M. (2021). The COVID-19 Pandemic and its Impact on the Professional Practice and Personal Wellbeing of Community Pharmacy Teams in the UK. Int. J. Pharm. Pract., riab062. doi:10.1093/ijpp/ riab062 importantly, although many kinds of popular medicines have been used in the management of COVID-19, so far only a few TCM preparations appear to have been tested systematically in RCTs, and even then these studies remain small and of uncertain quality. High quality clinical studies are urgently needed to appropriately guide evidence-informed approaches to incorporation of TMs in public health systems responses to COVID-19. Research using in silico or in vitro methods may have some values but the results need to be investigated further in vivo and clinically in order to allow an assessment of potential therapeutic benefits. Another limitation on the research on TMs against COVID-19 is the limited access to appropriate animal models, for example, transgenic hACE2 mice model (Bao et al., 2020) and cytokine storm syndrome mouse model induced by SARS-CoV-2 Spike protein (Gu et al., 2021). On the other hand, long COVID, the post-acute COVID-19 syndrome, has emerged as a major concern, which deserves further studies by using TMs (Adeloye et al., 2021). Overall, this research topic has been one of the first responses of the scientific communities globally to assess the potential of TM and here specifically TMs in addressing this major global health challenge. It has demonstrated the enormous importance and potential of TMs globally in response to the pandemic and the fast evolving scientific evidence base for some of these treatments. However, it also serves as a calling for a much more systematic study of TMs globally including, obviously, the need for major funding of this research, in order to be appropriately informed on this topic.

\section{AUTHOR CONTRIBUTIONS}

All authors edited MS, contributed to the RT as such and reviewed the MS at various stages.

Brendler, T., Al-Harrasi, A., Bauer, R., Gafner, S., Hardy, M. L., Heinrich, M., et al. (2021). Botanical Drugs and Supplements Affecting the Immune Response in the Time of COVID-19: Implications for Research and Clinical Practice. Phytother Res. 35 (6), 3013-3031. Epub 2020 Dec 29. PMID: 33373071. doi:10.1002/ptr.7008

Chen, R. H., Yang, L. J., Hamdoun, S., Chung, S. K., Lam, C. W., Zhang, K. X., et al. (2021). 1,2,3,4,6-Pentagalloyl Glucose, a RBD-ACE2 Binding Inhibitor to Prevent SARS-CoV-2 Infection. Front. Pharmacol. 12, 634176. doi:10.3389/ fphar.2021.634176

Fakhri, S., Piri, S., Majnooni, M. B., Farzaei, M. H., and Echeverría, J. (2020). Targeting Neurological Manifestations of Coronaviruses by Candidate Phytochemicals: A Mechanistic Approach. Front. Pharmacol. 11, 621099. doi:10.3389/fphar.2020.621099

Ge, C., and He, Y. (2020). In Silico Prediction of Molecular Targets of Astragaloside IV for Alleviation of COVID-19 Hyperinflammation by Systems Network Pharmacology and Bioinformatic Gene Expression Analysis. Front. Pharmacol. 11, 556984. doi:10.3389/fphar.2020.556984

Gu, T., Zhao, S., Jin, G., Song, M., Zhi, Y., Zhao, R., et al. (2021). Cytokine Signature Induced by SARS-CoV-2 Spike Protein in a Mouse Model. Front. Immunol. 11, 621441. doi:10.3389/fimmu.2020.621441

Heinrich, M. (2010). Ethnopharmacology in the 21st century - Grand Challenges. Front. Pharmacol. 1, 8. doi:10.3389/fphar.2010.00008

Jha, N. K., Sharma, C., Hashiesh, H. M., Arunachalam, S., Meeran, M. N., Javed, H., et al. (2021). $\beta$-Caryophyllene, A Natural Dietary CB2 Receptor Selective Cannabinoid $\mathrm{Can} \mathrm{Be}$ a Candidate to Target the Trinity of Infection, 
Immunity, and Inflammation in COVID-19. Front. Pharmacol. 12, 590201. doi:10.3389/fphar.2021.590201

Kalhori, M. R., Saadatpour, F., Arefian, E., Soleimani, M., Farzaei, M. H., Aneva, I. Y., et al. (2021). The Potential Therapeutic Effect of RNA Interference and Natural Products on COVID-19: A Review of the Coronaviruses Infection. Front. Pharmacol. 12, 616993. doi:10.3389/fphar.2021.616993

Kim, C. H. (2021). Anti-SARS-CoV-2 Natural Products as Potentially Therapeutic Agents. Front. Pharmacol. 12, 590509. doi:10.3389/fphar.2021.590509

Lem, F. F., Opook, F., Lee, D. J. H., Chee, F. T., Lawson, F. P., and Chin, S. N. (2020). Molecular Mechanism of Action of Repurposed Drugs and Traditional Chinese Medicine Used for the Treatment of Patients Infected with COVID-19: A Systematic Scoping Review. Front. Pharmacol. 11, 585331. doi:10.3389/ fphar.2020.585331

Li, Q., Bai, C., Yang, R., Xing, W., Pang, X., Wu, S., et al. (2020). Deciphering the Pharmacological Mechanisms of Ma Xing Shi Gan Decoction against COVID19 through Integrating Network Pharmacology and Experimental Exploration. Front. Pharmacol. 11, 581691. doi:10.3389/fphar.2020.581691

Lim, X. Y., Teh, B. P., and Tan, T. Y. C. (2021). Medicinal Plants in COVID-19: Potential and Limitations. Front. Pharmacol. 12, 611408. doi:10.3389/ fphar.2021.611408

Liu, T., Guo, Y., Zhao, J., He, S., Bai, Y., Wang, N., et al. (2020). Systems Pharmacology and Verification of ShenFuHuang Formula in Zebrafish Model Reveal Multi-Scale Treatment Strategy for Septic Syndrome in COVID-19. Front. Pharmacol. 11, 584057. doi:10.3389/fphar.2020.584057

Luo, C. H., Ma, L. L., Liu, H. M., Liao, W., Xu, R. C., Ci, Z. M., et al. (2020). Research Progress on Main Symptoms of Novel Coronavirus Pneumonia Improved by Traditional Chinese Medicine. Front. Pharmacol. 11, 556885. doi:10.3389/ fphar.2020.556885

Ma, L. L., Liu, H. M., Luo, C. H., He, Y. N., Wang, F., Huang, H. Z., et al. (2021). Fever and Antipyretic Supported by Traditional Chinese Medicine: A MultiPathway Regulation. Front. Pharmacol. 12, 583279. doi:10.3389/ fphar.2021.583279

Majnooni, M. B., Fakhri, S., Shokoohinia, Y., Kiyani, N., Stage, K., Mohammadi, P., et al. (2020). Phytochemicals: Potential Therapeutic Interventions against Coronavirus-Associated Lung Injury. Front. Pharmacol. 11, 588467. doi:10.3389/fphar.2020.588467

Mandal, A., Jha, A. K., and Hazra, B. (2021). Plant Products as Inhibitors of Coronavirus 3CL Protease. Front. Pharmacol. 12, 583387. doi:10.3389/ fphar.2021.583387

Peter, A. E., Sandeep, B. V., Rao, B. G., and Kalpana, V. L. (2020). Calming the Storm: Natural Immunosuppressants as Adjuvants to Target the Cytokine Storm in COVID19. Front. Pharmacol. 11, 583777. doi:10.3389/fphar.2020.583777

Qiu, Q., Huang, Y., Liu, X., Huang, F., Li, X., Cui, L., et al. (2020). Potential Therapeutic Effect of Traditional Chinese Medicine on Coronavirus Disease 2019: A Review. Front. Pharmacol. 11, 570893. doi:10.3389/fphar.2020.570893

Remali, J., and Aizat, W. M. (2020). A Review on Plant Bioactive Compounds and Their Modes of Action against Coronavirus Infection. Front. Pharmacol. 11, 589044. doi:10.3389/fphar.2020.589044

Ren, W., Ma, Y., Wang, R., Liang, P., Sun, Q., Pu, Q., et al. (2021). Research Advance on Qingfei Paidu Decoction in Prescription Principle, Mechanism Analysis and Clinical Application. Front. Pharmacol. 11, 589714. doi:10.3389/ fphar.2020.589714
Silveira, D., Prieto-Garcia, J. M., Boylan, F., Estrada, O., Fonseca-Bazzo, Y. M., Jamal, C. M., et al. (2020). COVID-19: Is There Evidence for the Use of Herbal Medicines as Adjuvant Symptomatic Therapy? Front. Pharmacol. 11, 581840. doi:10.3389/fphar.2020.581840

Smith, T., Majid, F., Eckl, V., and Morton Reynolds, C. (2021). Herbal Supplement Sales in US Increase by Record-Breaking $17.3 \%$ in 2020. Herbal Gram. (131), 52-65. Available: http://herbalgram.org/resources/herbalgram/issues/131/ table-of-contents/hg131-mkrpt/.

Spicer, D. (2021). Pilot Trial of XFBD, a TCM, in Persons with COVID-19. Available at: https://clinicaltrials.gov/ct2/show/NCT04810689 August 17, 2021).Assessed at

Verma, S., Twilley, D., Esmear, T., Oosthuizen, C. B., Reid, A. M., Nel, M., et al. (2020). Anti-SARS-CoV Natural Products with the Potential to Inhibit SARSCoV-2 (COVID-19). Front. Pharmacol. 11, 561334. doi:10.3389/ fphar.2020.561334

Wu, C. Y., Lin, Y. S., Yang, Y. H., Shu, L. H., Cheng, Y. C., and Liu, H. T. (2020). Potential Simultaneous Inhibitors of Angiotensin-Converting Enzyme 2 and Transmembrane Protease, Serine 2. Front. Pharmacol. 11, 584158. doi:10.3389/ fphar.2020.584158

Wu, J., Sun, B., Hou, L., Guan, F., Wang, L., Cheng, P., et al. (2020). Prospective: Evolution of Chinese Medicine to Treat COVID-19 Patients in China. Front. Pharmacol. 11, 615287. doi:10.3389/fphar.2020.615287

Yang, C. W., Lee, Y. Z., Hsu, H. Y., Jan, J. T., Lin, Y. L., Chang, S. Y., et al. (2020). Inhibition of SARS-CoV-2 by Highly Potent Broad-Spectrum Anti-coronaviral Tylophorine-Based Derivatives. Front. Pharmacol. 11, 606097. doi:10.3389/ fphar.2020.606097

Zhang, X. R., Li, T. N., Ren, Y. Y., Zeng, Y. J., Lv, H. Y., Wang, J., et al. (2020). The Important Role of Volatile Components from a Traditional Chinese Medicine Dayuan-Yin against the COVID-19 Pandemic. Front. Pharmacol. 11, 583651. doi:10.3389/fphar.2020.583651

Zhou, Z., Gao, N., Wang, Y., Chang, P., Tong, Y., and Fu, S. (2020). Clinical Studies on the Treatment of Novel Coronavirus Pneumonia with Traditional Chinese Medicine-A Literature Analysis. Front. Pharmacol. 11, 560448. doi:10.3389/ fphar.2020.560448

Conflict of Interest: The authors declare that the research was conducted in the absence of any commercial or financial relationships that could be construed as a potential conflict of interest.

Publisher's Note: All claims expressed in this article are solely those of the authors and do not necessarily represent those of their affiliated organizations, or those of the publisher, the editors and the reviewers. Any product that may be evaluated in this article, or claim that may be made by its manufacturer, is not guaranteed or endorsed by the publisher.

Copyright $\odot 2021$ Wang, Andrade-Cetto, Echeverria, Wardle, Yen and Heinrich. This is an open-access article distributed under the terms of the Creative Commons Attribution License (CC BY). The use, distribution or reproduction in other forums is permitted, provided the original author(s) and the copyright owner(s) are credited and that the original publication in this journal is cited, in accordance with accepted academic practice. No use, distribution or reproduction is permitted which does not comply with these terms. 